\title{
Cost-effectiveness of WHO Problem Management Plus for adults with mood and anxiety disorders in a post-conflict area of Pakistan: randomised controlled trial
}

Syed Usman Hamdani, Zill-e-Huma, Atif Rahman, Duolao Wang, Tao Chen, Mark van Ommeren, Dan Chisholm and Saeed Farooq

\section{Background}

With the development of evidence-based interventions for treatment of priority mental health conditions in humanitarian settings, it is important to establish the cost-effectiveness of such interventions to enable their scale-up.

\begin{abstract}
Aims
To evaluate the cost-effectiveness of the Problem Management Plus (PM+) intervention compared with enhanced usual care (EUC) for common mental disorders in primary healthcare in Peshawar, Pakistan. Trial registration ACTRN12614001235695 (anzctr.org.au).
\end{abstract}

\section{Method}

We randomly allocated 346 participants to either $\mathrm{PM}+(n=172)$ or EUC $(n=174)$. Effectiveness was measured using the Hospital Anxiety and Depression Scale (HADS) at 3 months post-intervention. Cost-effectiveness analysis was performed as incremental costs (measured in Pakistani rupees, PKR) per unit change in anxiety, depression and functioning scores.

\section{Results}

The total cost of delivering PM+ per participant was estimated at PKR 16967 (US\$163.14) using an international trainer and supervisor, and PKR 3645 (US\$35.04) employing a local trainer. The mean cost per unit score improvement in anxiety and depression symptoms on the HADS was PKR 2957 (95\% Cl 22624029) (US\$28) with an international trainer/supervisor and PKR
588 (95\% Cl 434-820) (US\$6) with a local trainer/supervisor. The mean incremental cost-effectiveness ratio (ICER) to successfully treat a case of depression (PHQ-9 $\geq 10$ ) using an international supervisor was PKR 53770 (95\% Cl 39394-77 399) (US\$517), compared with PKR 10705 (95\% Cl 7731-15 627) (US\$102.93) using a local supervisor.

\section{Conclusions}

The PM+ intervention was more effective but also more costly than EUC in reducing symptoms of anxiety, depression and improving functioning in adults impaired by psychological distress in a post-conflict setting of Pakistan.

\section{Keywords}

Cost-effectiveness; lay health worker; humanitarian setting; Problem Management Plus; common mental disorder (CMD).

\section{Copyright and usage}

(C) The Author(s), 2020. Published by Cambridge University Press on behalf of the Royal College of Psychiatrists. This is an Open Access article, distributed under the terms of the Creative Commons Attribution-NonCommercial-ShareAlike licence (http://creativecommons.org/licenses/by-nc-sa/4.0/), which permits non-commercial re-use, distribution, and reproduction in any medium, provided the same Creative Commons licence is included and the original work is properly cited. The written permission of Cambridge University Press must be obtained for commercial re-use.
Mental health problems cause a significant burden of disease in lowand middle-income countries (LMICs), yet the documented 'mental health treatment gap' is up to $90 \%{ }^{1-3}$ The need for mental health services is much greater in populations affected by humanitarian crises. More than 135 million people are in need of humanitarian assistance owing to ongoing humanitarian crises and conflicts globally. ${ }^{4}$ A systematic review and meta-analysis of mental health outcomes in populations affected by conflict and displacements showed that mood and anxiety disorders were common, with rates of $17.3 \%$ for depression and $15.4 \%$ for post-traumatic stress disorder. ${ }^{5}$ Epidemiological studies from areas affected by humanitarian crises in Pakistan found high rates of psychological distress in these populations. One study reported rates as high as $38-65 \%$ for psychological distress in women. ${ }^{6,7}$ The majority of people have no access to mental health services in such settings. ${ }^{6}$ Over the past decade, significant progress has been made in terms of availability of evidence-based mental health intervention packages for populations affected by humanitarian crises. ${ }^{8}$ However, sustainability and scalability of such psychological interventions remain a challenge in populations affected by humanitarian crises in lowresource settings globally. ${ }^{9}$

We developed and tested a brief, multicomponent behavioural intervention, Problem Management Plus (PM+), delivered by lay health workers for common mental disorders in conflict-affected settings. The intervention was effective for treating the symptoms of common mental disorders in a post-conflict setting in Pakistan. The trial protocol and results of pilot and definitive clinical trials have been published. ${ }^{10-12}$

In the present study, we conduct an economic evaluation alongside the randomised controlled trial to assess the cost-effectiveness of this intervention in order to inform policy and implementation in routine clinical practice.

\section{Method}

\section{Study site and participants}

Participants were 346 primary care attendees with high levels of psychological distress (score $>2$ on the General Health Questionnaire (GHQ-12) ${ }^{13}$ ) and functional impairment (score $>16$ on the 12 -item version of the World Health Organization Disability Assessment Schedule 2.0 (WHODAS 2.0) ${ }^{14}$ ). The participants were individually randomised in a 1:1 ratio to either the intervention arm, i.e. PM+ along with enhanced usual care (EUC) $(n=172)$ or the control arm, i.e. EUC only $(n=174)$. The study was approved locally by the Institutional Review and Ethics Board of 
the Postgraduate Medical Institute, Lady Reading Hospital, Peshawar, and by the WHO Research Ethics Review Committee. Written informed consent was obtained from all study participants.

\section{The intervention}

Participants in the intervention arm received a brief multicomponent intervention called Problem Management Plus $(\mathrm{PM}+)$ in addition to EUC. ${ }^{15} \mathrm{PM}+$ is transdiagnostic as it applies the same underlying principles across mental disorders, without tailoring the protocol to specific diagnoses. ${ }^{16} \mathrm{PM}+$ is based on wellestablished principles of problem-solving and behavioural techniques. It is designed to be used with adults experiencing common mental health problems (e.g. anxiety, stress, depression and grief) only. It is not suitable for the treatment of severe mental health problems (including psychosis or risk of suicide). Both an individual and a group version of the intervention exists. The current study involves the individual version.

$\mathrm{PM}+$ consists of 5 weekly face-to-face sessions of $90 \mathrm{~min}$ each, delivered by trained lay health workers. The intervention is composed of four core strategies (stress management; managing problems; 'get going, keep doing' (behavioural activation); and strengthening social support), introduced sequentially in the intervention sessions. In the last session, all the strategies are reviewed with an emphasis on using these strategies for self-management in the future and to prevent relapse.

Training and supervision followed a cascade model. An international trainer trained local trainers during a 6-day training workshop. Training consisted of intervention delivery, training and supervision skills. Local trainers cascaded the training to lay health workers (with 12-16 years of education) in an 8-day training. Lay health workers were provided weekly supervision by local trainers/supervisors (hereafter, local supervisor), who were in turn supervised monthly by the international trainer/supervisor (hereafter, international supervisor) via video conference for $2-3 \mathrm{~h}$. PM+ is available in Urdu and English on the WHO website. ${ }^{17}$ Further details of the intervention are described elsewhere. ${ }^{15}$

\section{Enhanced usual care}

The participants in both the intervention arm and the control arm received enhanced usual care (EUC). The care was enhanced as the primary healthcare physicians in the participating primary healthcare centres received a 5-day training in the management of common mental disorders in primary healthcare settings. The training was reinforced through a 1-day refresher course for the physicians. The study participants in both arms were able to seek other healthcare services from their primary healthcare physicians.

\section{Data collection}

\section{Health outcomes}

The outcomes were measured at baseline and 3 months post-intervention. The cost-effectiveness analysis was performed as incremental costs per unit change in anxiety, depression and functioning scores. The primary outcome was change in symptoms of anxiety and depression measured with the Hospital Anxiety and Depression Scale (HADS) ${ }^{18,19}$ Severity of symptoms was measured using the HADS anxiety subscale (7 items; possible score range, $0-21$ ) and depression subscale (7 items; possible score range, $0-21$ ). Higher scores indicate greater anxiety and/or depression. Secondary outcomes were functional impairment and presence of depressive disorders. The 12-item WHODAS 20 was used to assess functional impairment. The polytomous scoring algorithm was used to transform the functional impairment scores on a scale of $1-100 .{ }^{14}$ Presence of depressive disorder was measured using the 9-item Patient Health Questionnaire (PHQ-9). ${ }^{20}$ Other secondary outcome measures included the PTSD Checklist for DSM-5 (PCL-5) ${ }^{21}$ results of which appear in the supplementary material, available at http://doi.org/10.1192/bjp.2020.138.

\section{Health resource use profiling}

The data on health resource use were collected using the Client Services Receipt Inventory (CSRI), ${ }^{22}$ which records patients' contact with outpatient services (i.e. mental health specialists, general physicians, traditional healers, community health workers, etc.), in-patient (hospital admissions) services and out-of-pocket costs associated with travel, medications and tests/investigations during the preceding recall period. A section on seeking religious help and retreats was added to adapt the tool for use in the local population. Study participants self-reported their healthcare utilisation, medication use and out-of-pocket expenditures on the $\mathrm{CSRI}^{22}$ at baseline and 3 months post-intervention.

\section{Cost measurement and analysis}

Economic analysis was conducted primarily from a health system perspective, consisting of (a) costs incurred over the trial period in the delivery of the intervention itself, (b) use of other healthcare and related services by study participants, including religious help and retreats, and (c) patient and family costs (such as number of days with reduced working hours, informal caregiving time by relatives or friends, as well as travel costs and time spent travelling to or waiting for consultations). No discounting of costs was applied, since the study was performed within 1 year.

Intervention costs included: costs for the intervention adaptation workshops; translations of the $\mathrm{PM}+$ manual and training materials; printing of adapted training manuals; and staff recruitment, training and supervision. Supervision costs included time spent by the master trainer, supervisors, transport costs for fieldwork supervision and costs of all other resources used.

To estimate the cost of intervention delivery, we evaluated unit cost per minute of healthcare providers' time, including the international trainer/supervisor, local supervisors, lay health workers and physicians. To calculate the total cost of intervention delivery, the unit cost per minute was multiplied by the total estimated time spent by each healthcare provider with the participants. We calculated the cost of intervention delivery by the international trainer/supervisor and modelled the cost for a local supervisor as a potentially more sustainable way to support task-shifting in low-resource settings. Costs of the intervention were calculated by multiplying the total contact time (number of minutes) a participant in the intervention arm had with a lay health worker by the per-minute cost of the lay health workers' time and the costs spent on travelling by lay health workers (unit cost calculations are provided in the supplementary material).

Calculation of these intervention costs as well, as the cost of contacts with a range of formal healthcare providers, was facilitated by the use of a simplified costing template for unit cost calculations reported in health economic evaluation of mental health services. ${ }^{23}$ Unit cost templates accounted for the costs of salaries of staff employed in the provision of intervention delivery (including trainer, supervisors, lay health workers and primary healthcare staff), facility operating costs where the service was provided, overhead costs relating to the provision of service (personnel, finance, etc.) and the capital costs of the facility where the intervention was provided (land, buildings, etc.). Sources of data for these variables included public health system financial records and the project's financial records. All costs were calculated in Pakistani rupees (PKR) and are reported in Pakistani rupees and US dollars for the year 2016, when the study was implemented (at an exchange rate of US\$1 = PKR 104; www.ceicdata.com/en). No adjustment was made for purchasing power parity (PPP), since the focus of interest was the actual resource costs incurred in the study country (rather than a comparison with other countries, whereby 
differences in the relative price of goods and services would need to be taken into account).

\section{Statistical analysis}

The mean and standard deviation for the total cost were calculated using a generalised linear regression model with gamma distribution after adjustment for baseline total cost. The group difference and its $95 \%$ confidence interval was also calculated. ${ }^{24}$ The incremental cost-effectiveness ratio (ICER) was calculated as the additional costs of the intervention divided by the change in HADS anxiety, HADS depression, HADS total, PHQ-9 and 12-item WHODAS 20 scores related to the intervention. The confidence intervals for the ICER were estimated by non-parametric bootstrapping. The bootstrap technique sampled with replacement from the original observed pairs of costs and effects, maintaining the correlation structure between costs and benefits, to create a new data-set with 1000 observations. For each bootstrap resample, an estimate of differential total mean costs and the expected mean effectiveness was calculated. ${ }^{25}$ The $95 \%$ confidence intervals for the differential estimates were derived from the calculated 2.5th and 97.5th percentiles. We plotted cost-effectiveness acceptability curves ${ }^{26}$ to evaluate the probability of the $\mathrm{PM}+$ intervention being cost-effective at increasing monetary values, representing willingness-to-pay thresholds for the intervention from policy makers' perspective. ${ }^{27}$ For the effectiveness data, we used linear mixed models to study treatment effects as indicated in our main trial report, ${ }^{12}$ which allowed the number of observations to vary at random between participants and effectively handles missing data. ${ }^{28}$ At 3-month follow-up, $14 \%$ of cost data was missing for medicines, complementary medicines, seeking retreats and religious help, and out-patient services. Summary statistics for each specific cost were presented without imputation but the total costs were calculated assuming missing data as 0 in a conservative way. ${ }^{25}$

\section{Results}

As reported in the clinical effectiveness evaluation, ${ }^{12}$ mean combined depression and anxiety symptom scores on the HADS were significantly lower at 3 months post-intervention (adjusted mean difference -5.75 ; $95 \%$ CI -7.21 to -4.29 ). Similarly, functional impairment significantly improved (adjusted mean difference $-4.17 ; 95 \%$ CI -5.84 to -2.51 ) on the 12-item WHODAS in the intervention arm compared with the EUC arm. At baseline, the depression rate was $94.2 \%$ in the intervention arm and $89.4 \%$ in the EUC arm. At the end of the 3-month follow-up period, the intervention arm had significantly lower rates of depression $(26.9 \%)$ compared with the EUC arm (58.9\%) (risk difference -31.98 ; 95\% CI -41.03 to -22.94).

\section{Costs}

No significant difference in the cost of other healthcare services accessed by study participants was observed between treatment and control groups, with the exception of religious help and retreats. The mental health condition of the majority of trial participants did not result in reduction in their or their family members' or friends' usual work/activities (Table 1). Table 2 presents summary statistics and cost results from the mixed-model analysis.

With an international trainer/supervisor, the total cost of delivering PM+ per participant was PKR 16967 (US\$163.14). The total intervention arm costs $(\mathrm{PM}+$ costs plus cost of services accessed by the intervention arm as part of EUC) were PKR17 473 (s.d. $=912$ ) or US\$168. The cost of EUC (treatment as usual plus cost of services accessed by control arm participants) was PKR 848 (s.d. $=1734$ ) or US $\$ 8.15$ (Table 2).

Substituting the cost of an international trainer/supervisor with that of a local trainer would substantially decrease intervention costs. The total cost of delivering PM+ using a local trainer/supervisor was estimated to be PKR 3645 (US\$35.04). This would be PKR 729 (US\$7.00) per session. The total cost of delivering the intervention (with a local trainer/supervisor) plus EUC in the intervention arm would be PKR 4151 (s.d. =912) or US\$40.

\section{Cost-effectiveness}

Incremental cost-effectiveness ratios (ICERs) indicate that the intervention was both more effective and costlier than EUC for all the health outcomes studied (Table 3). Analysis was conducted to evaluate the cost-effectiveness of the PM+ intervention in two scenarios:

\begin{tabular}{|c|c|c|c|c|c|c|c|}
\hline & \multirow[b]{2}{*}{ Group } & \multicolumn{3}{|c|}{ Baseline } & \multicolumn{3}{|c|}{3 months post-intervention } \\
\hline & & $n(\%)$ & $\begin{array}{c}\text { Number of visits } \\
\text { Mean (s.d.) }\end{array}$ & $\begin{array}{l}\text { Visit duration, min } \\
\text { Mean (s.d.) }\end{array}$ & $n(\%)$ & $\begin{array}{l}\text { Number of visits } \\
\text { Mean (s.d.) }\end{array}$ & $\begin{array}{l}\text { Visit duration, } \min \\
\text { Mean (s.d.) }\end{array}$ \\
\hline \multicolumn{8}{|l|}{ Out-patient services } \\
\hline \multirow{2}{*}{ Traditional healer } & $\mathrm{PM}+$ with EUC & $40(12.0)$ & $4.03(3.83)$ & $26(28.04)$ & $9(3.0)$ & $3.38(3.15)$ & $6.54(16.75)$ \\
\hline & EUC alone & $50(15.1)$ & $3.47(2.50)$ & $25.13(25.17)$ & $19(6.3)$ & $2.26(0.80)$ & $5.00(9.71)$ \\
\hline \multirow[t]{2}{*}{ Mental health professional } & $\mathrm{PM}+$ with EUC & $91(29.4)$ & $4.36(4.73)$ & $15.82(13.08)$ & $80(26.9)$ & $3.61(1.87)$ & $16.73(9.39)$ \\
\hline & EUC alone & $76(24.5)$ & $3.09(2.38)$ & $17.21(15.28)$ & $98(33.0)$ & $3.08(1.49)$ & $17.48(9.98)$ \\
\hline \multirow[t]{2}{*}{ Medical doctor } & $\mathrm{PM}+$ with EUC & 57 (18.4) & $2.98(2.20)$ & $17.93(24.58)$ & 39 (13.1) & $2.17(1.72)$ & $11.79(7.23)$ \\
\hline & EUC alone & $54(17.5)$ & $3.56(4.23)$ & $23.29(29.42)$ & $37(12.5)$ & $1.94(1.01)$ & $16.67(12.50)$ \\
\hline \multirow[t]{2}{*}{ Community health worker } & $\mathrm{PM}+$ with EUC & $56(16.9)$ & $4.90(5.72)$ & - & $25(8.2)$ & $4.0(2.58)$ & - \\
\hline & EUC alone & 54 (16.3) & $3.87(4.33)$ & - & $25(8.2)$ & $2.54(1.53)$ & - \\
\hline \multirow[t]{2}{*}{ Any other services } & $\mathrm{PM}+$ with EUC & $11(3.4)$ & $2.56(2.87)$ & - & $6(2.0)$ & $1.20(0.44)$ & - \\
\hline & EUC alone & $8(2.5)$ & $1.38(0.91)$ & - & $3(1.0)$ & $2.0(1.73)$ & - \\
\hline \multirow[t]{2}{*}{ Religious help and retreats } & $\mathrm{PM}+$ with EUC & $37(11)$ & $6.86(11.90)$ & - & $7(2.4)$ & $3.71(5.02)$ & - \\
\hline & EUC alone & 45 (13.4) & $3.33(4.84)$ & - & $14(4.8)$ & $3.15(2.99)$ & - \\
\hline \multirow[t]{2}{*}{ In-patient services } & $\mathrm{PM}+$ with EUC & $8(2.3)$ & $3.29(2.43)^{\mathrm{a}}$ & - & 7 (2.3) & $7.20(12.75)^{\mathrm{a}}$ & - \\
\hline & EUC alone & $13(3.8)$ & $3.91(4.10)^{\mathrm{a}}$ & - & $8(2.6)$ & $2.5(0.53)^{a}$ & - \\
\hline \multirow{2}{*}{$\begin{array}{l}\text { Reduced usual work/activities } \\
\text { due to health condition } \\
\text { (participant or family } \\
\text { member) }\end{array}$} & $\mathrm{PM}+$ with EUC & $6(1.8)$ & $21.33(15.01)^{b}$ & - & $0(0.0)$ & - & - \\
\hline & EUC alone & $1(0.3)$ & - & - & $1(0.3)$ & - & - \\
\hline
\end{tabular}


Table 2 Cost of health services accessed by participants (out-patient, in-patient, drugs/medication, and complimentary medicines and religious retreats) by trial $\mathrm{arm}^{\mathrm{a}}$

\begin{tabular}{|c|c|c|c|c|c|c|c|}
\hline \multirow[b]{2}{*}{ Service } & \multirow[b]{2}{*}{ Time point } & \multicolumn{2}{|c|}{ PM+ with EUC $(N=172)$} & \multicolumn{2}{|c|}{ EUC alone $(N=174)$} & \multirow[b]{2}{*}{$\begin{array}{c}\text { Difference in least } \\
\text { squares mean }(95 \% \mathrm{Cl})\end{array}$} & \multirow[b]{2}{*}{$P$} \\
\hline & & $n$ & $\begin{array}{c}\text { Cost, PKR } \\
\text { Mean (s.d.) }\end{array}$ & $n$ & $\begin{array}{c}\text { Cost, PKR } \\
\text { Mean (s.d.) }\end{array}$ & & \\
\hline \multirow[t]{3}{*}{ Out-patient care } & Pre-treatment & 106 & 2641 (14 946) & 95 & 727 (1161) & & \\
\hline & Follow-up & 73 & $485(651)$ & 72 & $667(1033)$ & $-182(-465$ to 101$)$ & 0.206 \\
\hline & Change since baseline & 49 & $743(2751)$ & 41 & $305(984)$ & 437 (-462 to 1281$)$ & 0.336 \\
\hline \multirow[t]{3}{*}{ In-patient care } & Pre-treatment & 170 & $135(929)$ & 172 & $273(1545)$ & & \\
\hline & Follow-up & 142 & 49 (344) & 155 & $171(1056)$ & $-122(-304$ to 61$)$ & 0.191 \\
\hline & Change since baseline & 140 & $114(866)$ & 153 & 108 (1953) & $6(-337$ to 349$)$ & 0.971 \\
\hline \multirow[t]{3}{*}{ Drugs/medications } & Pre-treatment & 158 & 736 (1364) & 159 & 725 (1232) & & \\
\hline & Follow-up & 132 & $277(650)$ & 149 & $228(461)$ & 50 (-82 to 181) & 0.458 \\
\hline & Change since baseline & 124 & 378 (1314) & 136 & 496 (1341) & -118 ( -442 to 207$)$ & 0.477 \\
\hline \multirow[t]{3}{*}{ Complimentary medicines } & Pre-treatment & 168 & $124(624)$ & 167 & $110(945)$ & & \\
\hline & Follow-up & 139 & $10(88.14)$ & 156 & $3(40)$ & 7 (-9 to 22$)$ & 0.393 \\
\hline & Change since baseline & 136 & $55(456)$ & 150 & 115 (998) & $-60(-244$ to 123$)$ & 0.518 \\
\hline \multirow[t]{3}{*}{ Religious retreats } & Pre-treatment & 167 & 390 (2208) & 165 & $674(3773)$ & & \\
\hline & Follow-up & 136 & $4(43)$ & 154 & $131(655)$ & $-127(-238$ to -17$)$ & 0.024 \\
\hline & Change since baseline & 131 & 432 (2451) & 145 & $626(4080)$ & $-193(-983$ to 596$)$ & 0.638 \\
\hline \multirow{3}{*}{ Total cost of all services } & Pre-treatment & 172 & 3145 (14 302) & 174 & 2445 (6053) & & \\
\hline & Follow-up & 145 & $601(694)$ & 159 & 848 (1734) & $-247(-568$ to 73$)$ & 0.130 \\
\hline & Change since baseline & 145 & 2746 (15 491) & 159 & 1714 (6632) & 1032 (-1709 to 3774$)$ & 0.444 \\
\hline \multirow{2}{*}{\multicolumn{2}{|c|}{$\begin{array}{l}\text { Total cost of interventions with } \mathrm{PM}+\text { provided by } \\
\text { international specialist supervisor } \\
\text { Total cost of interventions with PM+ provided by local } \\
\text { specialist supervisor }{ }^{\mathrm{C}}\end{array}$}} & 172 & 17473 (912) & 159 & 848 (1734) & 16625 (16329 to 16922$)$ & $<0.0001$ \\
\hline & & 172 & 4151 (912) & 159 & 848 (1734) & 3303 (3007 to 3600) & $<0.0001$ \\
\hline \multicolumn{8}{|c|}{$\begin{array}{l}\text { PM+, Problem Management Plus; EUC, enhanced usual care. } \\
\text { a. The data were collected using the Client tervices Receipt Inventory at baseline and at the 3-month post-intervention follow-up assessment. Costs are shown in Pakistan rupees (PKR); the } \\
2016 \text { exchange rate was } 1 U S \$=104 \text { PKR. } \\
\text { b. Intervention costs plus cost of services. The cost of the PM+ intervention using an international supervisor is PKR } 16967 . \\
\text { c. Intervention costs plus cost of services. The cost of the PM+ intervention using a local supervisor is PKR } 3645 \text {. }\end{array}$} \\
\hline
\end{tabular}

(a) PM+ delivery by lay health workers supervised by an international trainer/supervisor (as observed in the trial) and (b) PM+ delivery by lay health workers supervised by a local supervisor. The second scenario will be the case for scale-up of the intervention package in realworld settings. The additional costs associated with the intervention led to a relative improvement in outcomes. For example, the mean cost per unit score improvement in anxiety and depression on the HADS was PKR 2957 (95\% CI 2262-4029) or US\$28 with an international trainer/supervisor. This would be PKR 588 (95\% CI 434820 ) or US\$6 with a local trainer/supervisor; with an international supervisor, each 1-point improvement on the WHODAS cost PKR 4097 (95\% CI 2978-6046) or US\$40, whereas with a local supervisor it was estimated to cost PKR 815 (95\% CI 576-1225) or US\$8. We plotted 1000 resampled estimates of costs and outcomes on a costeffectiveness plane for the primary and secondary outcomes. The results show that all the resampled estimates fall in the upper-right quadrant, i.e. $\mathrm{PM}+$ is 'more effective but costlier' in all of the resampled estimates.

The mean ICER to successfully treat a case of depression (PHQ-9 cut-off $\geq 10)$ using an international supervisor was PKR 53770 (95\% CI 39 394-77 399) (US\$517), compared with PKR 10705 (95\% CI
7731-15 627) (US\$102.93) using a local supervisor. ICERs for other outcome measures are compared in Table 3.

The cost-effectiveness acceptability curves for the PM+ intervention in relation to outcomes on the HADS (total score) and 12-item WHODAS with an international specialist supervisor are shown in Figs 1 (a) and 2(a). The intervention has more than $90 \%$ probability of being cost-effective compared with EUC above a willingness-topay threshold of PKR 7000 (US\$67) for a one-point improvement in depression and anxiety (HADS total score) (Fig. 1(a)) and PKR 6000 (US\$57) for a one-point improvement in functioning (WHODAS) using international supervisors (Fig. 2(a)). These thresholds would be reduced by $80 \%$ using local supervisors (Figs 1(b) and 2(b)).

\section{Discussion}

\section{Main findings}

Our results show that the $\mathrm{PM}+$ intervention $(\mathrm{PM}+$ together with enhanced usual care, EUC) is more effective and more costly than EUC alone in reducing symptoms of anxiety and depression. Although there is inevitable uncertainty regarding point estimates,

Table 3 Incremental cost-effectiveness ratios (ICERs) ${ }^{\text {a }}$ for the PM+ intervention at 3 months post-intervention for international versus local supervisors

\begin{tabular}{|c|c|c|c|c|}
\hline & \multicolumn{2}{|c|}{ International specialist supervisor } & \multicolumn{2}{|c|}{ Local specialist supervisor } \\
\hline & Mean ICER, PKR & $95 \% \mathrm{Cl}^{\mathrm{b}}$ & Mean ICER, PKR & $95 \% \mathrm{Cl}^{\mathrm{b}}$ \\
\hline HADS Anxiety & 6172.99 & $4575.49-8787.73$ & 1228.91 & $882.86-1796.12$ \\
\hline HADS Depression & 5704.27 & $4384.51-7651.85$ & 1135.81 & $849.23-1561.68$ \\
\hline HADS Total & 2957.45 & $2261.64-4029.00$ & 588.82 & $434.01-820.27$ \\
\hline WHO DAS & 4096.51 & $2978.13-6045.66$ & 815.89 & $575.80-1225.10$ \\
\hline Depression caseness & 53769.91 & $39393.57-77398.62$ & 10705.35 & $7730.95-15627$ \\
\hline \multicolumn{5}{|c|}{$\begin{array}{l}\text { PM+ intervention, Problem Management Plus with enhanced usual care; HADS, Hospital Anxiety and Depression Scale (subscale score range: } 0-21 \text {; higher scores indicate elevated anxiety } \\
\text { or depression, respectively); WHODAS, World Health Organization Disability Assessment Schedule } 2.0 \text { (12-item version, total score range: } 0-48 \text {; higher scores indicate more severe } \\
\text { impairment); depression caseness, defined as a Patient Health Questionnaire score } \geq 10 \text {. } \\
\text { a. Costs are shown in Pakistan rupees (PKR); the } 2016 \text { exchange rate was } 1 \text { US } \$=104 \text { PKR. Costs were estimated after adjusting several baseline variables (baseline total cost, age, gender, } \\
\text { occupation, marital status). } \\
\text { b. Confidence intervals were estimated using non-parametric bootstrapping with } 1000 \text { resamples. }\end{array}$} \\
\hline
\end{tabular}



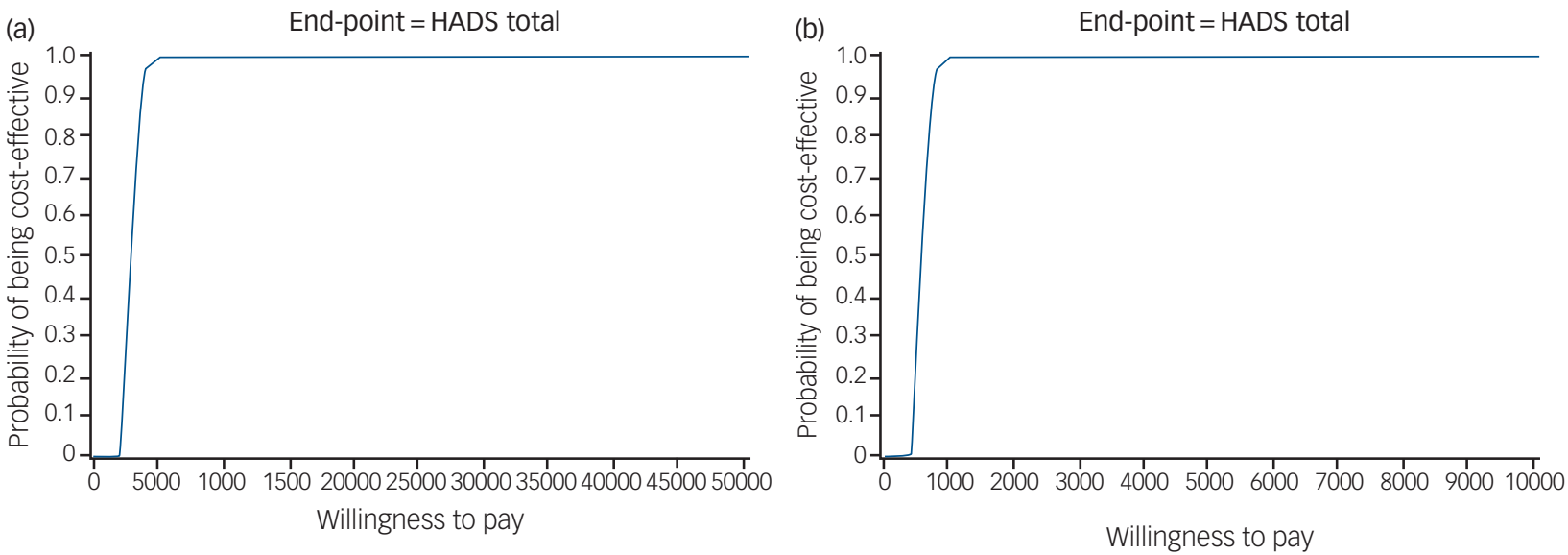

Fig. 1 Cost-effectiveness acceptability curves for Problem Management Plus $(\mathrm{PM}+)$ in relation to improvement in Hospital Anxiety and Depression Scale (HADS) total score at 3-month follow-up (end-point). (a) PM+ using an international supervisor. (b) PM+ using a local supervisor. Costs are shown in Pakistan rupees (PKR); the 2016 exchange rate was 1 US $\$=104$ PKR.

our analysis has shown that even at very modest levels of willingness to pay for a one-point improvement in symptoms or functioning outcomes there is at least a $90 \%$ probability of this intervention being a cost-effective use of resources compared with EUC. We concluded that the value is 'modest' because that amount is equivalent to, for example, less than $10 \%$ of the minimum monthly wage in Pakistan in 2017. ${ }^{29}$ These findings are consistent with evidence from LMICs on the cost-effectiveness of a task-shifting approach to delivering psychological interventions for the treatment of common mental disorders compared with EUC delivered by primary healthcare physicians. ${ }^{30,31}$ With the current model of training and supervision by an international trainer/supervisor, the intervention was five times more costly for treating one person with depression, compared with modelled costs of training and supervision by local trainers. This emphasises the need to build the capacity of a local mental health workforce. $^{32}$

\section{Comparison with the literature}

The resources, capacity and infrastructure for mental health services research, including health economic evaluations alongside randomised controlled trials, are limited in the humanitarian settings of LMICs. ${ }^{33}$ This is one of the very few studies to evaluate the cost-effectiveness of a psychological intervention in a humanitarian setting. There are only a few published studies on the costeffectiveness of task-shifting interventions in global mental health. Araya et al (2006) evaluated the incremental cost-effectiveness of a stepped-care multicomponent programme for the treatment of women with depression in primary care in Chile. The stepped-care programme was more effective and costlier than usual care (an extra US $\$ 0.75$ per depression-free day). ${ }^{34}$ Buttorff et al (2012) conducted an economic evaluation of a task-shifting intervention for the treatment of depressive and anxiety disorders in primary care settings in India. They concluded that the use of lay health workers in the treatment of common mental disorders in public primary care facilities was not only cost-effective but also cost-saving. The mean health system cost per person recovered at the end of follow-up was US\$128 (95\% CI 105-157) in the intervention arm and US\$149 (95\% CI 131-169) in the control arm. ${ }^{30}$ Other similar studies of psychological interventions delivered by lay health counsellors in India ${ }^{31}$ have replicated the findings of cost-effectiveness of task-shifting interventions for
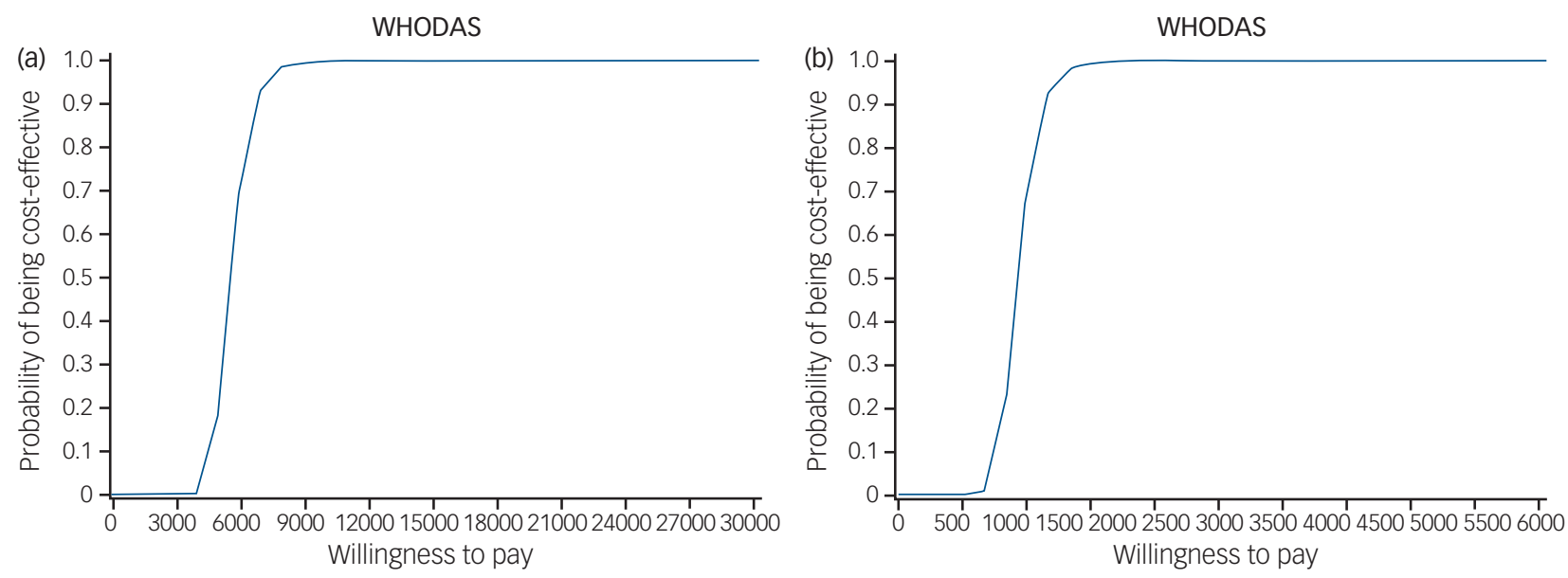

Fig. 2 Cost-effectiveness acceptability curves for Problem Management Plus (PM+) in relation to improvement in score on the 12-item version of the World Health Organization Disability Assessment Schedule 2.0 (WHODAS 20) at 3-month follow-up (end-point). (a) PM+ using an international supervisor. (b) PM+ using a local supervisor. Costs are shown in Pakistan rupees (PKR); the 2016 exchange rate was 1 US $\$=104$ PKR. 
treating depression and alcohol problems in primary care settings. Sikander et al (2019) evaluated the cost-effectiveness of a peervolunteer-delivered cognitive-behavioural intervention for postnatal depression compared with EUC in community settings of rural Pakistan. The intervention was costlier than EUC but was effective in reducing the severity of post-natal depression (the cost per unit improvement in PHQ-9 score was US\$15.50 (95\% CI 9.59-21.61) for the whole study period). The intervention had a $98 \%$ probability of being cost-effective above a willingness-to-pay threshold of US\$60 per unit of improvement on PHQ-9 score compared with EUC. ${ }^{35}$ Although it is difficult to compare the results of cost-effectiveness evaluations across studies (owing to differences in analytical approaches, treatment conditions and different outcome measures), the results of these studies demonstrate the cost-effectiveness of brief psychological interventions using a task-shifting approach.

During humanitarian crises, healthcare systems tend to be overwhelmed, human resources are overstretched and access to specialists for referral and support is limited. It is therefore important to determine how interventions with proven efficacy can be scaled up in a cost-effective way. ${ }^{36}$ Our study and evidence from the literature support the effectiveness of implementation strategies such as task-shifting and transdiagnostic approaches to bridge the treatment gap for mental health problems in low-resource settings. With the increased availability of evidence-based psychological intervention packages, further health economic evaluations are needed to inform the resource needs to scale up evidence-based care for mental illness.

\section{Limitations}

A limitation of the cost-effectiveness approach used in our study is that the results are limited to direct healthcare costs and healthrelated outcomes of the PM+ intervention, and do not extend to the wider economic or social value of investing in mental health, which may be quite significant in a humanitarian context. Future health economic evaluations of global mental health will benefit by integrating the opportunity and time cost of lay health workers and non-specialists. The added value that results from such task-sharing implementation strategies in terms of empowerment, opportunities and career growth for the nonspecialist healthcare workforce as well as the increase in treatment coverage for priority mental health conditions will also need to be accounted for in future studies. We did not make any adjustment for purchasing power parity (PPP), since the focus of this study was the actual resource costs incurred in the study country. However, for the purpose of international comparison, the PPP adjusted total intervention costs of PM+ were I $\$ 546$ per participant. Estimated costs of delivering $\mathrm{PM}+$ using a local trainer in Pakistan would be I $\$ 114$ per participant. Another limitation of our study is that we estimated costs per point reduction in symptoms of anxiety and depression and cost per person recovered from depression, which limits the ability to compare results with other interventional studies on the basis of cost-utility measures (quality-adjusted life years, QALYs). Future studies may use change in health outcomes that are easily interpretable and meaningful enough for policy makers to make decisions, and should also collect data on population-based health-state preference scores that would enable the calculation of QALYs.

Syed Usman Hamdani (D), PhD, Institute of Life and Human Sciences, University of Liverpool, UK; and Human Development Research Foundation, Islamabad, Pakistan; Zill-e-Huma, MPH, Human Development Research Foundation, Islamabad, Pakistan; Atif Rahman, PhD, Institute of Life and Human Sciences, University of Liverpool, UK; Duolao Wang, PhD, Liverpool School of Tropical Medicine, UK; Tao Chen, PhD, Liverpool School of Tropical Medicine, UK; Mark van Ommeren, PhD, Department of Mental Health and Substance Abuse, World Health Organization, Geneva, Switzerland;
Dan Chisholm, PhD, WHO Regional Office for Europe, Copenhagen, Denmark Saeed Farooq, PhD, Lady Reading Hospital, Peshawar, Pakistan; and School of Primary, Community and Social Care, Keele University, UK.

Correspondence: Syed Usman Hamdani. Email: s.u.hamdani@liverpool.ac.uk

First received 9 Sep 2019, final revision 10 Jun 2020, accepted 20 Jun 2020

\section{Supplementary material}

Supplementary material is available online at http://doi.org/10.1192/bjp.2020.138.

\section{Data availability}

Data are available from the authors on request.

\section{Acknowledgements}

We thank the project staff in the Department of Psychiatry, Lady Reading Hospital, Peshawar, and the Human Development Research Foundation (HDRF), Islamabad, Pakistan, for their contributions; the primary healthcare staff and physicians for their support in the conduct of the study: and the participants and their families for their voluntary participation. We would like to specially thank Dr Victoria Baranov (senior lecturer in economics, University of Melbourne, Australia) for sharing her insights in revising the manuscript for resubmission.

\section{Author contributions}

S.U.H., Z.e.H., T.C. and D.W. had full access to all of the data in the study and take responsibility for the integrity of the data and the accuracy of the data analysis. Concept and design: S.U.H. A.R., S.F. D.C. M. . O.; acquisition, analysis or interpretation of data: S.U.H. Z. .H. D.W. S.F. M.v.O., D.C.; drafting of the manuscript: S.U.H., Z.e.H., A.R., M.v.O., D.C., S.F.; critical revision of the manuscript for important intellectual content: S.U.H., Z.e.H., A.R., S.F., D.C., T.C., D.W., M.V.O.; statistical analysis: S.U.H., Z.e.H., D.C., T.C., D.W.; obtained funding: M.V.O., A.R., S.U.H.; administrative, technical, or material support: S.U.H., Z.e.H., A.R., S.F., M.v.O.; study supervision: S.U.H., A.R., S.F., M.V.O.

\section{Funding}

This work was supported by the Enhanced Learning and Research for Humanitarian Assistance (Elhra) Research for Health in Humanitarian Crises (R2HC) initiative, funded by the UK Department for International Development and the Wellcome Trust. The funders had no role in the design and conduct of the study; collection, management, analysis, and interpretation data; preparation, review, or approval of the manuscript; and decision to submit the manuscript for publication.

\section{Declaration of interest}

M.v.O. and D.C. are staff members of the World Health Organization. The authors alone are responsible for the views expressed in this publication and they do not necessarily represent the decisions, policy or views of the World Health Organization.

ICMJE forms are in the supplementary material, available online at https://doi.org/10.1192/ bjp.2020.138

\section{References}

1 Demyttenaere K, Bruffaerts R, Posada-Villa J, Gasquet I, Kovess V, Lepine J, et al. Prevalence, severity, and unmet need for treatment of mental disorders in the World Health Organization World Mental Health Surveys. JAMA 2004; 291: $2581-90$

2 Saxena S, Thornicroft G, Knapp M, Whiteford H. Resources for mental health: scarcity, inequity, and inefficiency. Lancet 2007; 370: 878-89.

3 Thornicroft G. Most people with mental illness are not treated. Lancet 2007 370: $807-8$.

4 United Nations Office for the Coordination of Humanitarian Affairs. Global Humanitarian Overview 2018. OCHA, 2017 (https://reliefweb.int/sites/reliefweb.int/files/resources/GHO2018.PDF).

5 Steel Z, Chey T, Silove D, Marnane C, Bryant RA, Van Ommeren M. Association of torture and other potentially traumatic events with mental health outcomes among populations exposed to mass conflict and displacement: a systematic review and meta-analysis. JAMA 2009; 302: 537-49.

6 Roberts B, Browne J. A systematic review of factors influencing the psychological health of conflict-affected populations in low-and middle-income countries. Glob Public Health 2011; 6: 814-29. 
7 Tol WA, Barbui C, Van Ommeren M. Management of acute stress, PTSD, and bereavement: WHO recommendations. JAMA 2013; 310: 477-8.

8 Bangpan M, Lambert F, Chiumento A, Dickson K. The Impact of Mental Health and Psychosocial Support Programmes for Populations Affected by Humanitarian Emergencies: A Systematic Review Protocol. Oxfam, 2015.

9 Allden $\mathrm{K}$, Jones $\mathrm{L}$, Weissbecker I, Wessells M, Bolton P, Betancourt T, et al. Mental health and psychosocial support in crisis and conflict: report of the Mental Health Working Group. Prehosp Disaster Med 2009; 24(suppl 2): s217-27.

10 Rahman A, Riaz N, Dawson KS, Usman Hamdani S, Chiumento A, Sijbrandij M et al. Problem Management Plus (PM+): pilot trial of a WHO transdiagnostic psychological intervention in conflict-affected Pakistan. World Psychiatry 2016; 15: 182-3.

11 Sijbrandij M, Farooq S, Bryant RA, Dawson K, Hamdani SU, Chiumento A, et al. Problem Management Plus (PM+) for common mental disorders in a humanitarian setting in Pakistan; study protocol for a randomised controlled trial (RCT). BMC PSychiatry 2015; 15(1): 232.

12 Rahman A, Hamdani SU, Awan NR, Bryant RA, Dawson KS, Khan MF, et al. Effect of a multicomponent behavioral intervention in adults impaired by psychological distress in a conflict-affected area of Pakistan: a randomized clinical trial. JAMA 2016; 316: 2609-17.

13 Minhas F, Mubbashar M. Validation of General Health Questionnaire (GHQ-12) in primary care settings of Pakistan. J Coll Physicians Surg Pak 1996; 6: 133-6.

14 Üstün TB, Kostanjsek N, Chatterji S, Rehm J. Syntax for automatic computation of overall score using SPSS. In Measuring Health and Disability: Manual for WHO Disability Assessment Schedule WHODAS 2.0: 59-62. WHO, 2010.

15 Dawson KS, Bryant RA, Harper M, Kuowei Tay A, Rahman A, Schafer A, et al. Problem Management Plus (PM+): a WHO transdiagnostic psychological intervention for common mental health problems. World Psychiatry 2015; 14: 354-7.

16 McEvoy PM, Nathan P, Norton PJ. Efficacy of transdiagnostic treatments: A review of published outcome studies and future research directions. J Cogn Psychol 2009; 23: 20-33.

17 World Health Organization. Problem Management Plus (PM+): Individual Psychological Help for Adults Impaired by Distress in Communities Exposed to Adversity (Generic Field-Trial Version 1.0). WHO, 2016.

18 Zigmond AS, Snaith RP. The hospital anxiety and depression scale. Acta Psychiatr Scand 1983; 67: 361-70.

19 Mumford D, Tareen I, Bajwa M, Bhatti M, Karim R. The translation and evaluation of an Urdu version of the Hospital Anxiety and Depression Scale. Acta Psychiatr Scand 1991; 83: 81-5.

20 Kroenke K, Spitzer RL, Williams JB. The PHQ-9: validity of a brief depression severity measure. J Gen Intern Med 2001; 16: 606-13.

21 Weathers FW, Litz BT, Keane TM, Palmieri PA, Marx BP, Schnurr PP. The PTSD Checklist for DSM-5 (PCL-5). National Center for PTSD, 2013.

22 Chisholm D, Knapp MRJ, Knudsen HC, Amaddeo F, Gaite L, Van Wijngaarden B, et al. Client Socio-Demographic and Service Receipt Inventory-European Version: development of an instrument for international research: EPSILON Study 5. Br J Psychiatry 2000; 177(suppl 39): s28-33.
23 Patel V, Weiss HA, Chowdhary N, Naik S, Pednekar S, Chatterjee S, et al. Effectiveness of an intervention led by lay health counsellors for depressive and anxiety disorders in primary care in Goa, India (MANAS): a cluster randomised controlled trial. Lancet 2010; 376: 2086-95.

24 McCrone P, Knapp M, Proudfoot J, Ryden C, Cavanagh K, Shapiro DA, et al. Cost-effectiveness of computerised cognitive-behavioural therapy for anxiety and depression in primary care: randomised controlled trial. Eur J Psychotraumatol 2004; 185: 55-62.

25 Khan I. Design \& Analysis of Clinical Trials for Economic Evaluation \& Reimbursement: An Applied Approach using SAS \& STATA. Chapman \& Hall/ CRC Press, 2015

26 Baltussen RMPM, Hutubessy RCW, Evans DB, Murray CJM. Uncertainty in costeffectiveness analysis: probabilistic uncertainty analysis and stochastic league tables. Int J Technol Assess Health Care 2002; 18: 112-9.

27 Fenwick E, Marshall DA, Levy AR, Nichol G. Using and interpreting cost-effectiveness acceptability curves: an example using data from a trial of management strategies for atrial fibrillation. BMC Health Serv Res 2006; 6(1): 52.

28 Little RJA, Rubin DB. Statistical Analysis with Missing Data (3rd edn). John Wiley \& Sons, 2019.

29 Govt. of Pakistan Finance Division Islamabad: Federal Budget, 2017-18 (http:// finance.gov.pk/budget/Federal_Budget_Press_Brief_2017_18.pdf).

30 Buttorff C, Hock RS, Weiss HA, Naik S, Araya R, Kirkwood BR, et al. Economic evaluation of a task-shifting intervention for common mental disorders in India. Bull World Health Organ 2012; 90: 813-21.

31 Weobong B, Weiss HA, McDaid D, Singla DR, Hollon SD, Nadkarni A, et al Sustained effectiveness and cost-effectiveness of the Healthy Activity Programme, a brief psychological treatment for depression delivered by lay counsellors in primary care: 12-month follow-up of a randomised controlled trial. PLOS Med 2017; 14(9): e1002385.

32 Murray LK, Dorsey S, Bolton P, Jordans MJ, Rahman A, Bass J, et al. Building capacity in mental health interventions in low resource countries: an apprenticeship model for training local providers. Int J Ment Health Syst 2011; 5(1): 30.

33 Levin C, Chisholm D. Cost-effectiveness and affordability of interventions, policies, and platforms for the prevention and treatment of mental, neurological, and substance use disorders. In Mental, Neurological, and Substance Use Disorders (Disease Control Priorities, 3rd edn, vol. 4) (eds V Patel, D Chisholm, T Dua, R Laxminarayan, ME Medina-Mora). World Bank, 2015.

34 Araya R, Flynn T, Rojas G, Fritsch R, Simon G. Cost-effectiveness of a primary care treatment program for depression in low-income women in Santiago, Chile. Am J Psychiatry 2006; 163: 1379-87.

35 Sikander S, Ahmad I, Atif N, Zaidi A, Vanobberghen F, Weiss HA, et al. Delivering the Thinking Healthy Programme for perinatal depression through volunteer peers: a cluster randomised controlled trial in Pakistan. Lancet Psychiatry 2019; 6: 128-39.

36 Ventevogel $P$, van Ommeren M, Schilperoord M, Saxena S. Improving mental health care in humanitarian emergencies. J Bull World Health Organ 2015; 93 : 666-666A. 